\title{
L'APPLICAZIONE DELLE NORME UE SULLA CONCORRENZA AL SETTORE SPAZIALE EUROPEO: IL PROCUREMENT PER IL GNSS GALILEO COME CASE STUDY.
}

\author{
LA APLICACIÓN DE LAS NORMAS UE SOBRE COMPETENCIA \\ AL SECTOR ESPACIAL EUROPEO: EL PROCUREMENT PARA \\ EL GNSS GALILEO COMO CASE STUDY.
}

\author{
THE APPLICATION OF EU COMPETITION RULES TO THE \\ EUROPEAN OUTERSPACE SECTOR : THE PROCUREMENT \\ PROCESS FOR THE GNSS GALILEO AS CASE STUDY.
}

\begin{abstract}
VALERIO MAIONE*
Sommario: I. - PREMESSA. II. - IL CONTESTO DI RIFERIMENTO. III. - LE PECULIARITÀ DELLA DISCIPLINA DELLA CONCORRENZA NELL' “OUTERSPACE» EUROPEO. IV. - LA DISCIPLINA DELLA CONCORRENZA TRA UE E STATI MEMBRI. V. LE AUTORIZZAZIONI E LE CONCESSIONI DI LICENZE SPAZIALI IN RAPPORTO AL DIRITTO DELLA CONCORRENZA. VI. - L'IMPATTO DEL SISTEMA GALILEO. VII. - LE NOVITÀ SPERIMENTATE PER IL PROCUREMENT DEL GNSS GALILEO. VIII. - CONCLUSIONI.

Sumario: I. - INTRODUCCIÓN. II. - EL MARCO EN REFERENCIA. III. - LAS PECULIARIDADES DEL DERECHO DE COMPETENCIA EN EL «OUTERSPACE» EUROPEO. IV. - LA NORMATIVA SOBRE COMPETENCIA ENTRE LA UE Y LOS ESTADOS MIEMBROS. V. - LAS AUTORIZACIONES Y LAS CONCESIONES DE LICENCIAS ESPACIALES RESPECTO A LA NORMATIVA DE COMPETENCIA. VI. - EL IMPACTO DEL SISTEMA GALILEO. VII. - LAS NOVEDADES EXPERIMENTADAS CON EL PROCUREMENT DEL GNSS GALILEO. VIII. CONCLUSIONES.

Summary: I. - INTRODUCTION. II. - FRAMEWORK. III. - THE CHARACTERISTICS OF COMPETITION LAW WITHIN THE EUROPEAN OUTERSPACE FRAMEWORK. IV. - THE COMPETITION RULES BETWEEN EU AND MEMBER STATES . V. - AUTHORIZATIONS AND LICENSING SCHEME WITHIN OUTERSPACE FRAMEWORK WITH REGARD TO COMPETITION LAW. VI. - THE IMPACT OF GALILEO SYSTEM . VII. - THE NEW EXPERIENCE FOR GNSS GALILEO PROCUREMENT. VIII. - CONCLUSIONS.
\end{abstract}

Resumen: El actual desarrollo de las competencias espaciales de la Unión Europea está produciendo la extensión de la aplicación de las normas sobre competencia de la UE a los contratos y concesiones en el ámbito del espacio ultraterrestre. Aunque este dato se refiera solo a los programas espaciales europeos más importantes, en particular el sistema GALILEO, representa la violación de un sector que las lobbies de las industrias espaciales de los Estados miembros de la ESA suelen defender. Este artículo presenta una reconstrucción de los diferentes sistemas de procurement de la ESA y la UE, delinenado sus cambios recientes, el acercamiento progresivo, sino también su inevitable incompatibilidad. El objetivo final de este trabajo es poner de relieve el

\footnotetext{
* Avvocato, Dottore di Ricerca e Cultore della Materia in Diritto internazionale e dell’Unione Europea presso l’Università degli Studi di Napoli “Parthenope” (Italia). E-mail: valerio.maione@uniparthenope.it.
} 
impacto actual y futuro del procedimiento de procurement creado para el sistema GALILEO de acuerdo con las normas sobre competencia de la UE, sugiriendo que pudiera no quedar un caso aislado, sino que a posteriori podrá revelarse un progreso más de la Comisión Europea en su finalidad de prevalecer en el cd. triangulo institucional de la gobernanza multinivel de el "outerspace” europeo.

Abstract: The current stage of EU competence on outerspace is producing the extension of the application of EU competition rules to procurement. Although this still concerns only major european space programs, in particular, the Galileo system symbolizes the violation of a matter particularly defended by the industry lobbies of the ESA Member States. This article analyzes different procurement systems within ESA and EU, their recent changes, their progressive approaching but also their incompatibility. The ultimate aim of this work is to highlight current and future impact of the ongoing experimentation with the procurement process for the Galileo system according to the EU antitrust rules, pointing out the possibility that it might be not an isolated case. On the contrary, a posteriori it might be read as further step in the progressive European Commission's will to prevail in the so called "institutional triangle” of the European multilevel governance on outerspace.

Sintesi: Lo sviluppo attuale delle competenze spaziali dell'Unione Europea sta producendo l'estensione dell'applicazione delle norme sulla concorrenza UE agli appalti ed alle concessioni in materia di spazio. Sebbene tale ultimo dato riguardi ancora soltanto i principali programmi spaziali europei, in particolare il sistema GALILEO, esso rappresenta la violazione di un ambito particolarmente difeso dalle lobbies delle industrie degli Stati membri ESA. Questo articolo presenta una ricostruzione dei differenti sistemi di procurement dell'ESA e dell'UE, delinenado le rispettive recenti modifiche, il loro progressivo avvicinamento ma anche la loro inelluttabile incompatibilità. Lo scopo ultimo di questo lavoro è quello di evidenziare l'impatto attuale e futuro della sperimentazione in corso con la procedura d'appalto per il sistema Galileo secondo la normativa antitrust UE, paventando la possibilità che non resti un caso isolato, bensì che possa rivelarsi a posteriori un ulteriore step del progressivo disegno della Commissione europea di prevalere nel $\mathrm{cd}$. triangolo istituzionale della governance multilivello dell’outerspace europeo.

Palabras clave: GNSS galileo; ESA; UE; competencia; procurement Keywords: GNSS Galileo; ESA; EU; competition; procurement.

Parole chiave: GNSS galileo; ESA; UE; concorrenza; procurement.

\section{PREMESSA}

Si può affermare che l'entrata in vigore del Trattato di Lisbona, estendendo la competenza $^{1}$ dell'Unione Europea al settore dello spazio ${ }^{2}$, ha creato un nuovo campo in

\footnotetext{
${ }^{1}$ Si ricorda qui che l'attribuzione della competenza spaziale dagli Stati membri all’Unione Europea trova il suo fondamento negli Artt. 4(3) e 189 del Trattato sul Funzionamento dell'Unione Europea. L'art. 189 va inquadrato peraltro nel titolo XIX ove si disciplinano "ricerca e sviluppo tecnologico e spazio" ed in particolare l'art. 179 con cui «L'Unione si propone l'obiettivo di rafforzare le sue basi scientifiche e
} 
cui si possono applicare le regole dell'Unione Europea in materia di concorrenza. Sebbene possa ritenersi azzardato ${ }^{3}$ ritenere che tale applicazione possa divenire la normale futura prassi nell'ambito del public procurement spaziale, essa per come si sta manifestando -mentre si sta scrivendo- per il procurement del sistema GALILEO ${ }^{4}$, rappresenta un rilevante elemento di novità ed un notevole ampliamento dell'ambito di attuazione del diritto europeo della concorrenza, posto che si tratta di un settore di mercato già oggi ampio e considerevole, ma soprattutto, (essendo strettamente legato alle tecnologie attualmente trainanti) in progressiva espansione.

Vale le pena ricordare che tale panorama è reso possibile dall'Accordo quadro ${ }^{5}$ ESA-UE che ha posto in essere un'architettura istituzionale e politica avanzata, in grado di garantire all'Unione una proficua collaborazione con l'Agenzia spaziale europea ${ }^{6}$, nonché, una netta demarcazione tra le attività spaziali svolte sotto l'egida dell'Unione Europea e quelle realizzate nell'ambito della competenza originaria dell' agenzia spaziale europea.

In questo scritto saranno esaminate le norme europee sulla concorrenza che attualmente si applicano alle attività spaziali, con un particolare focus sulla possibile interazione delle due Organizzazioni de quibus, dal punto di vista dei rispettivi

tecnologiche con la realizzazione di uno spazio europeo della ricerca nel quale i ricercatori, [... ] di favorire lo sviluppo della sua competitività, inclusa quella della sua industria [...]A tal fine essa incoraggia nell'insieme dell'Unione le imprese, comprese le piccole e le medie imprese, i centri di ricerca e le università [...] mirando soprattutto a permettere ai ricercatori di cooperare liberamente oltre le frontiere e alle imprese di sfruttare appieno le potenzialità del mercato interno grazie, in particolare, all'apertura degli appalti pubblici nazionali, alla definizione di norme comuni ed all'eliminazione degli ostacoli giuridici e fiscali a detta cooperazione».

${ }^{2}$ Il concetto di Politica Spaziale Europea è stato promosso per la prima volta in una Risoluzione del Parlamento europeo del 17 settembre 1981 (in GU C 260 del 12.10.1981, pag. 102), preceduta dalla più generica risoluzione del 25 aprile 1979 sulla partecipazione della Comunità alla ricerca spaziale (in GU C 127 del 21.5.1979, pag. 32).

${ }^{3}$ In ragione delle note divergenze, in tema di investimenti e di "giusto ritorno" per le imprese nazionali degli Stati membri, tra il sistema dell'Agenzia Spaziale Europea e le normative dell’Unione per il libero mercato, in termini di concorrenza, di appalti, di aiuti di Stato etc..

${ }^{4}$ Il sistema di radionavigazione satellitare globale (GNSS - Global Navigation Satellite System) già operativo ed in fase di ultimazione, risultato di uno dei programmi di bandiera dell'Unione Europea più prestigiosi, strategici ed economicamente impegnativi dell'Unione. Non a caso - si ricorda - una delle ragioni dell'introduzione della competenza spaziale dell'Unione mediante l'art. 189 TFUE è da ricercare, in misura rilevante, nell'alto profilo del progetto GNSS GALILEO (oltre che dell'altro programma spaziale di bandiera per l'osservazione terrestre “COPERNICUS” ex GMES Global Monitoring for Environment and Security) e dell'enorme indotto che genererà.

5 Accordo quadro "tra la Comunità europea e l’Agenzia spaziale europea” (firmato a Bruxelles il 21 novembre 2003 e ratificato con Decisione del Consiglio il 29 aprile 2004), volto principalmente alla realizzazione di una sistematica politica europea dello spazio in costante cooperazione tra le due organizzazioni (oggi tra ESA ed UE), fondata su basi normative più chiare e nel rispetto dei principi sulla “governance europea” espressi nel Libro Bianco del 2001 (COM (2001) 428 DEF)

${ }^{6}$ A.S.E., meglio nota con il più diffuso acronimo anglosassone E.S.A. La cooperazione tra Stati europei in ambito spaziale trae origine formalmente sin dai primi anni sessanta, dalle convenzioni istitutive dell'ELDO (Organizzazione Europea per lo Sviluppo e la Costruzione dei vettori spaziali) e dell'ESRO (Organizzazione Europea di Ricerca Spaziale) nel 1962, sino a giungere all'istituzione dell'Agenzia Spaziale Europea (ESA) nel 1975 (sostanzialmente, in sostituzione delle prime due) con l'obiettivo palese di dare luogo ad una politica spaziale europea. Essa pur essendo nata formalmente al di fuori delle Comunità europee, ha continuato a svilupparsi al di fuori del processo d'integrazione comunitaria ma in maniera in qualche modo parallela. 
programmi spaziali, da un lato, e delle regole del diritto della concorrenza, dall'altro, con particolare riferimento alla novità costituta dalla recente creazione, per GALILEO, del "GSO" (Galileo Satellite Operator) ${ }^{7}$ l'operatore per la gestione del "Procurement" spaziale europeo" (la gestione delle gare d'appalto e dei contratti per la fornitura di tutto quanto necessario alle missioni) che ha dovuto operare nelle piena applicazione del Diritto dell'Unione Europea in materia di concorrenza, appalti, concessioni, aiuti di Stato etc.

L'entrata in vigore del trattato di Lisbona ha segnato una biforcazione nel ruolo originale dell'ESA, quale iniziatore e gestore dei programmi spaziali europei o nazionali, dovendosi distinguere, in tal senso, tra i programmi spaziali che rientrano nelle competenze dell'Unione Europea e quelli che rientrano nelle competenze dell'ESA, e ciò in particolar modo dal punto di vista del diritto della concorrenza. Ciò in quanto l'UE e l'ESA sono due organizzazioni economiche e governative internazionali indipendenti, regolate da specifici e indipendenti Trattati costitutivi, i quali dettano norme sugli appalti che differiscono tra loro profondamente poiché riflettono divergenti presupposti ed obiettivi economici. L'approccio al diritto della concorrenza e agli appalti di ciascuna istituzione è appunto distinta, se non fondamentalmente opposta, così come i quadri finanziari delle singole istituzioni riflettono diversi obiettivi e approcci. Le differenti regole di bilancio di ciascuna istituzione impongono il rispetto di separate regole di procurement.

L'Unione Europea è ormai competente ad agire parallelamente ai suoi Stati membri in ambito spaziale, mentre, a livello inter-istituzionale, collabora con l'Agenzia spaziale europea $^{8}$. Per tale motivo, la disciplina della politica spaziale oggi richiede una maggiore precisione, dal momento che i progetti spaziali ormai rientrano sia nell'ambito delle normative dell'Unione sia in quello delle normative relative ai programmi spaziali dell' ESA. Infatti, se, da un lato, vi sono le norme UE in materia di appalti (sia di armonizzazione degli appalti tra gli Stati membri, sia di disciplina degli appalti centralizzati che ricadono sotto il sindacato europeo delle procedure finanziarie), dall'altro, vi sono la Convenzione ESA e le sue regole di bilancio ed anche le norme che regolano i progetti non europei dell'Agenzia spaziale europea.

A completare il quadro complessivo, vi è la tendenza all'armonizzazione del diritto nazionale spaziale, ossia il tentativo degli Stati membri di dotarsi di una normativa nazionale sullo spazio $^{9}$. La tendenza in parola riflette il crescente interesse da parte degli stati membri dell'Unione Europea e dell' ESA di dotarsi di un'infrastruttura normativa volta a favorire la costituzione di un contesto competitivo per il mercato

\footnotetext{
${ }^{7}$ Tale struttura di supporto va inquadrata nel contesto di una più ampia architettura di agenzie di supporto istituite per il raggiungimento degli obiettivi prefissati dall'UE con la creazione e per lo sviluppo dei programmi spaziali cd. “di bandiera”. Si pensi all’EUSC (European Union Satellite Center”) ma soprattutto, per ciò che qui più interessa, all' Agenzia del GNSS europeo, la quale ha sostituito la "Autorità di vigilanza del GNNS europeo" GSA (Galileo Satellite Authority) mantenendo il medesimo acronimo "GSA" che invece sta per "european Global navigation Satellite Agency" http://www.gsa.europa.eu/gsa/about-gsa e nel cui interno si colloca l'operato del GSO che gestisce appunto il procurement.

${ }^{8}$ Tale collaborazione istituzionale, come detto, ha avuto inizio per consentire progressi con il progetto Galileo. L'ESA, infatti - fondata nel 1975 - è l'unica agenzia spaziale in Europa ad avere le competenze tecniche necessarie per portare avanti questo progetto di alto profilo.

${ }^{9}$ M. Gerhard, in S. Hobe, B. Schmidt-Tedd e K.U. Schrogl (a cura di), in Cologne Commentary on Space Law, Vol. I (2009), n. VI, Heymanns Verlag, Köln, pp 120-122.
} 
spaziale in ascesa. Tuttavia, tale interesse si scontra con la circostanza che l'armonizzazione della legislazione nazionale sullo spazio non rientra nell'ambito delle attività dell'Unione Europea che sono soggette ai requisiti di armonizzazione del mercato unico. Quindi, la creazione di un comune framework per lo sviluppo di un "business dello spazio" in tutta l'Unione stenta a decollare, laddove, invece, un certo sviluppo della governance di settore conferirebbe un più elevato grado di coerenza tra la legislazione nazionale sullo spazio e quella UE, creando - finalmente - un quadro comune per le attività spaziali commerciali ed un mercato sempre più unificato. Una regolamentazione giuridica uniforme per i mercati spaziali nazionali favorirebbe il giro di affari già esistente e stimolerebbe quello con i paesi in via di sviluppo.

Tale panorama tuttavia non sarebbe completo se non si tenesse conto dei principi ai quali l'UE è vincolata in virtù del proprio status sui generis nell'ordinamento giuridico internazionale $^{10}$ e, quindi, degli effetti della divergente trasposizione, a livello nazionale, delle disposizioni dei Trattati di diritto internazionale sullo spazio ${ }^{11}$.

\section{IL CONTESTO DI RIFERIMENTO:}

\section{Il mercato dell'industria spaziale europea}

Come accennato, dal punto di vista del mercato, il settore spaziale è tradizionalmente suddiviso in pochissime grandi aziende ${ }^{12}$ alle quali si affiancano - in qualità di fornitori - molte piccole e medie imprese. L'avvento di nuovi mercati per le applicazioni spaziali in settori come l'osservazione terrestre, la navigazione e altri servizi di comunicazione hanno creato nuovi livelli di ingresso, in particolare per le piccole imprese, come il campo dello sviluppo di softwares. Questo fenomeno sta aprendo il mercato dell'Outerspace a nazioni tradizionalmente "non-spaziali" le quali si stanno concentrando su attività e servizi spaziali (che vanno dai servizi di registrazione

\footnotetext{
${ }^{10}$ L'esatta relazione tra la legge europea e il sistema giuridico internazionale è stata oggetto di numerosi dibattiti in seno alla Corte di Giustizia europea; si veda il Caso 22/70, Commissione vs. Consiglio (ERTA) del 1971; l'Opinione 2/91 del 1993 (ECR I-1061) e infine il Caso 26/62, Van Gend en Loos vs. Nederlandse Administratie Belastingen del 1963.

${ }^{11}$ Le funzioni attribuite allo Stato dalle disposizioni di cui all'articolo VI del Trattato sullo spazio extraatmosferico per l'istituzione di una unica licenza spaziale nazionale e di un regime di monitoraggio derivano in parte dal dovere, ai sensi del diritto internazionale, e ulteriormente ampliato in quel trattato all'articolo VII, di garantire la responsabilità nazionale per le operazioni spaziali commerciali . Cfr. Treaty on Principles Governing the Activities of States in the Exploration and Use of Outer Space, including the Moon and Other Celestial Bodies, Londra/Mosca/Washington, 27 Gennaio 1967. Tali doveri gravano anche sulle organizzazioni internazionali ai sensi dell'art. VII della "Registration Convention", che prevede che le organizzazioni internazionali possano fare dichiarazioni di accettazione dei termini della Convenzione. Alcuni Stati membri e le organizzazioni internazionali hanno assunto impegni formali nei confronti di responsabilità relative al piano di lancio di Ariane con questo obiettivo in mente. L'ESA ha formalmente comunicato l'accettazione dei suoi obblighi derivanti dai trattati internazionali di cui all'articolo XXII della convenzione sulla responsabilità. Cfr. Convenzione sulla Responsabilità internazionale per i danni causati dal lancio di oggetti nello spazio, Londra/Mosca/Washington, 29 marzo 1972.

${ }^{12}$ Come già descritto con alcuni esempi, molte industrie dello spazio in Europa sono il risultato di fusioni pan-europee tra ex monopolisti nazionali.
} 
per gli oggetti nello spazio, fino allo sviluppo di piccoli satelliti spaziali), senza tuttavia poter contare sulle classiche infrastrutture o risorse dell'industria spaziale. Tali sviluppi favoriscono la crescita del mercato, dal momento che consentono l'ingresso ad entità nuove e più piccole come subappaltatori e fornitori specializzati, a vari livelli, consentendo alle stesse di non limitare le proprie attività ai confini del mercato nazionale. Inoltre, in tutto il mondo l'industria spaziale è in gran parte sovvenzionata da programmi istituzionali che si traducono in finanziamenti ai programmi di ricerca e sviluppo $(R \& S)$ e nell'acquisto di prodotti e servizi spaziali. Le attività di R\&S europee rappresentano nel loro complesso il $10 \%$ circa del fatturato non consolidato del settore spaziale dell'UE. Nel contesto internazionale il finanziamento delle attività europee di R\&S appare relativamente ridotto. Ad esempio, circa il 25\% del bilancio spaziale civile statunitense è destinato alla R\&S. Inoltre, il budget civile pro capite della NASA da solo è superiore di circa quattro volte ai budget complessivi (budget nazionali, dell'ESA e dell'UE $/ 7^{\circ}$ programma quadro) del settore spaziale civile europeo. Il mercato istituzionale europeo è relativamente modesto - nel 2009 il budget degli Stati Uniti è stato di quasi dieci volte superiore al budget europeo - e molto frammentato, data la diversità dei soggetti pubblici interessati e delle loro diverse politiche industriali, non sempre coordinate.

\section{L'ambito intergovernativo dell'ESA}

L'industria mista pubblico-privata, la governance triangolare intergovernativosovranazionale $^{13}$ ed il complesso ruolo (tecnico-scientifico, governativo, gestionale e imprenditoriale) delle Agenzie spaziali nazionali, costituiscono peculiarità della struttura del settore spaziale. Queste, fanno sì che quando si affrontano gli aspetti commerciali di tale specifico settore industriale, si debba chiarire bene cosa si intende con il termine "procurement", rectius, per il caso specifico, di "public procurement".

Il public procurement può essere definito come l'acquisto regolamentato di beni o servizi da parte di - o in rappresentanza di - un'autorità pubblica, mediante fondi pubblici. Ciò presuppone che vi siano delle condizioni normativamente prestabilite per la stipulazione dei relativi contratti e che fondi pubblici entrino nel mercato privato influenzandolo. Conseguentemente, in dottrina ${ }^{14}$ è stato rilevato - peraltro con specifico riferimento al settore spaziale - che il confine tra public procurement e politica industriale è molto labile. Si tratta di un aspetto che nel settore spaziale assume una rilevanza tale da meritare autonome speculazioni scientifiche ${ }^{15}$ e conseguentemente

\footnotetext{
${ }^{13}$ L’Unione, l’ESA, gli Stati membri (e le agenzie spaziali nazionali di alcuni di essi) compongono un cd. “triangolo istituzionale” che vede l’UE “intromessa” nelle relazioni tra Stati membri e tra Stati membri ed ESA, costituendo un sistema di multilevel governance.

${ }^{14}$ R. Hansen, J. Wouters, Towards an EU policy for the space sector - Lessons from Galileo, in Space policy, Elsevier, 2012, n.28, pp 94-101, i quali rimarcano la necessità che la politca industriale europea dello spazio sia condivisa armoniosamente tra gli Stati e soprattutto venga supportata da strumenti normativi adeguati rispetto alle ambizioni della politica spaziale europea nel suo complesso

${ }^{15} \mathrm{Si}$ vedano, tra i vari, innazitutto S. Hobe, M. Hofmannova e J. Wouters, A Coherent european procuremnt law and policy for the space sector: toward a third way, in Cologne Studies in International and European Law, Lit Verlag, 2011, i quali nel richiamare l'importanza di assicurare nell'europa dello spazio sia coerenza che flessibilità nel procurement, profilano una terza via costituita dalla possibilità di introdurre talvolta dei cd.“ Extra-Procurement Instruments”; ancora, Ioannis Petrou,The Euopean Space
} 
impone nell'economia del presente lavoro di limitarsi ad un inquadramento strettamente funzionale all'argomento principale.

E' stato rilevato come il forte richiamo alla necessità di escludere, nell'accordo quadro ESA-UE, qualsiasi forma di applicazione della politica del cd. "ritorno geografico" (o "giusto ritorno"), sia già la testimonianza del contrasto di fondo che soggiace al sistema di procurement del settore spaziale ${ }^{16}$. E' - peraltro - diffusa l'opinione che questo specifico settore meriterebbe la possibilità di un trattamento differenziato, in termini di elasticità rispetto alle regole dell'UE sulla concorrenza e sul mercato. Ciò in ragione dell'alto tasso di tecnologia e degli alti costi per l'accesso allo spazio (specie con riferimento ai capitali necessari nelle lunghe fasi di iniziale sviluppo dei progetti spaziali), fattori in re ipsa potenzialmente in contrasto con i menzionati principi dell'UE. Basti pensare che questi possono costituire barriere all'entrata per nuovi attori, sia pubblici che privati, specie se si considera che le poche opportunità di realizzare economie di scala nel settore spaziale europeo afferiscono prevalentemente ad un mercato istituzionale in cui l'ESA (ed il relativo entourage di imprese in vario modo legate ai suoi Stati membri) ${ }^{17}$ vanta un ruolo dominante, sia come acquirente che come primo partner dei produttori ${ }^{18}$.

Appare opportuno al riguardo richiamare quanta importanza sia conferita, nella convenzione istitutiva dell'ESA, all'obiettivo del perseguimento di una politica industriale $^{19}$, come si evince dagli obiettivi sanciti all'art. 7. La dottrina ${ }^{20}$ ha infatti criticamente dedotto dalla lettura di tale articolo che la previsione alla lettera "d" risulti subordinata rispetto alle altre tre del medesimo articolo laddove si subordina l'adozione di "procedure di gare in condizioni di libera concorrenza" alla "compatibilità con gli altri citati obiettivi di politica industriale”. Si evince chiaramente che l'obiettivo della libera concorrenza è gerarchicamente subordinato rispetto agli altri ${ }^{21}$ (incluso quelli che - ai sensi dell'ultimo capoverso del medesimo articolo, possono essere nel tempo introdotti dal Consiglio dell’ESA mediante delibera all’unanimità).

\section{L'ambito del mercato regolamentato dell'UE}

Agency's procurement system: a critical assessment, in Public Contract Law Journal 2007-2008, n.37 pp. 197-205.

${ }^{16}$ Si veda la lettera c dell'art. 7 della Convenzione ESA, di seguito riportato, laddove indica quale terzo obiettivo della politica industriale «Ensure that all Member States participate in an equitable manner» ed aggiunge «having regard to their financial contribution».

17 Oppure, si pensi alle problematiche connesse alla circostanza che le differenti imprese nazionali “capocommessa” (ossia, la capofila delle imprese coinvolte nel contratto di fornitura) producono dei vettori che vengono verificati, integrati ed ultimati da Arianespace secondo le specifiche esigenze delle differenti delle missioni. Orbene, tali imprese non solo spesso sono a partecipazione statale - peraltro partecipazione spesso rappresentata dalle stesse agenzie nazionali coinvolte nella ricerca e sviluppo - ma sono al contempo sia fornitori che azionisti della medesima Arianespace.

${ }^{18}$ In ultimo, anche la caratteristica duale delle tecnologie spaziali (ossia che contempla le applicazioni militari) incide molto sull’ostracismo dei governi nei confronti di nuovi attori.

${ }^{19}$ Dato non scontato se si tiene in conto che l'ESA deriva dalla fusione di due Organizzazioni (l'ESRO e l'ELDO) una più improntata alla ricerca e l'altra più improntata ai programmi esecutivi.

${ }^{20}$ R. Hansen, J. Wouters, “Towards an EU policy for the space sector”, in http://www.kuleuven.be, 2015, working paper n. 149., http://ghum.kuleuven.be/ggs/wp149-hansen-woutersx.pdf.

${ }^{21}$ Così, S. Hobe, M. Hofmannova e J. Wouters, "A coherent european procurement law and policy for the space sector”, en id., cit. nota 15. 
Si ricorda brevemente che il diritto della concorrenza prevede due forme di controllo regolamentare: quello relativo all'ingresso nel mercato, disciplinato dal diritto delle intese ${ }^{22}$, e quello sulla concorrenza sleale, relativo al controllo del comportamento della società una volta attiva sul mercato. Le regole comunitarie sulla concorrenza sin da principio hanno mirato ad eliminare le distorsioni del mercato provocate da accordi in grado di produrre una limitazione della libera concorrenza sia a livello verticale che orizzontale ${ }^{23}$.

Come è noto l'attuale disciplina dei comportamenti anticoncorrenziali è contenuta negli articoli 101 e 102 (accanto a norme in materia di aiuti di Stato ai sensi dell'articolo 107) del Trattato sul funzionamento dell'Unione Europea (TFUE). Inoltre, le regole di concorrenza dell'UE si applicano anche alle imprese pubbliche e imprese con diritti speciali o esclusivi (articolo 106 TFUE). Di conseguenza può affermarsi - ed è ciò che più interessa rispetto al settore esaminato nel presente lavoro - che la concorrenza sleale vietata dalle norme del Trattato riguarda sia le imprese private che quelle pubbliche ${ }^{24}$. Per dare ancor di più il senso di quanto non sia scontata e non sia di poco momento la recente applicazione delle norme UE sulla concorrenza nel settore spaziale per il sistema Galileo, appare utile riportare testualmente il testo dell'art. 101 c.3 (neretto e sottolineature sono aggiunte) laddove nell'elencare le "intese" per cui «le disposizioni del paragrafo 1 possono essere dichiarate inapplicabili» fa salve appunto le opere:

- «che contribuiscano a migliorare la produzione o la distribuzione dei prodotti o a promuovere il progresso tecnico o economico, pur riservando agli utilizzatori una congrua parte dell'utile che ne deriva, ed evitando di:

a) imporre alle imprese interessate restrizioni che non siano indispensabili per raggiungere tali obiettivi;

b) dare a tali imprese la possibilità di eliminare la concorrenza per una parte sostanziale dei prodotti di cui trattasi.»

Orbene, la crescita dei mercati spaziali commerciali potrebbe vedere l'applicazione di queste norme alle nuove attività, ad esempio, nelle menzionate nuove applicazioni spaziali a valle, in particolare nello sviluppo del settore dell'informazione.

Quanto sopra appare ancor più evidente, se si pensa che, con la crescita del commercio spaziale, il ruolo delle Agenzie spaziali nazionali potrebbe ulteriormente sbilanciarsi verso il business, finendo per connotarle in modo prevalentetal senso e - finanche farle divenire degli attori in concorrenza nel settore, più palesemente di quanto non lo

\footnotetext{
${ }^{22}$ Si veda P. Trepte, “Public Procurement in the EU, a Practioner's Guidebook”, Oxford, 2a ed., 2007; Korah V., "An introductory Guide to EC Competition Law and Practice”, Oxford and Portland, 9th ed, , Hart, 2007.

${ }^{23}$ Il primo tipo di accordo può verificarsi tra la fase di produzione e quella di distribuzione, e il secondo, nel caso in cui delle imprese operano negli stessi mercati e allo stesso livello possono, al fine di condurre importanti ricerche e sviluppo e / o trasferimento di tecnologie, cooperare o anche occupare una posizione dominante su tale mercato particolare, bloccando così l'ingresso di nuovi operatori.

${ }^{24}$ L'articolo 101 del TFUE vieta gli accordi a livello sia verticale che orizzontale, che siano progettati per avere l'effetto di limitare il commercio tra gli Stati membri. L'articolo 102 del TFUE vieta l'abuso di posizione dominante sul mercato rilevante. Tuttavia, come è noto, eccezioni individuali o per categoria si applicano ai sensi dell'articolo 101 c.3, in particolare agli accordi di fornitura e di distribuzione, nonché di ricerca e sviluppo e sugli accordi di specializzazione. Questi normalmente ricadrebbero nell'ambito del divieto di cui all'articolo 101 c.1, ma possono comunque essere considerati leciti grazie all'effetto di cui beneficia un settore di mercato con determinate caratteristiche, come quello spaziale.
} 
siano già. Il mercato dei dati sullo spazio, in effetti, sta già portando spin-off e nuovi campi di attività in settori che tradizionalmente rientravano in ambiti sottoposti a sovranità statale.

\section{LE PECULIARITÀ DELLA DISCIPLINA DELLA CONCORRENZA NELL” “OUTERSPACE” EUROPEO}

Sotto il versante della concorrenza, il settore spaziale rappresenta un caso particolare, in quanto i finanziamenti pubblici che normalmente sono stanziati con accordi particolari rendono gli appalti pubblici una parte essenziale del processo concorrenziale. Il diritto della concorrenza, com'è noto, opera contro qualsiasi concentrazione del mercato, diretto o indiretto o restrizioni sleali della concorrenza da parte di una o più imprese. Il mercato spaziale, però, copre alcuni appalti militari e di difesa, permettendo di attuare delle parziali deroghe a beneficio di quelle imprese già attive in questo settore, le quali potranno sostenere il contraccolpo della tratteggiata evoluzione del mercato. Queste infatti potranno godere ancora della rendita di posizione derivante dalla pregressa partecipazione alle iniziative spaziali nazionali e/o programmi di difesa, con diverse fonti di finanziamento europeo o nazionale, a seconda di progetti di natura civile o militare. Simile considerazione - anche in chiave critica - può farsi anche con riferimento alla valutazione dei vantaggi competitivi rappresentati dalla fisiologica posizione dominante di queste stesse aziende storiche, nella partecipazione alle procedure di appalto competitive, che le favorisce nell'ottenere lo status di "primo contraente" o "capocommessa" e - quindi - nel continuare ad occupare una posizione privilegiata. L'acquisizione di know-how e di posizioni di mercato, infatti, rimane una preoccupazione costante del diritto della concorrenza.

Tuttavia, gli appalti per i contratti militari o di governo, se di dominio esclusivamente nazionale o intra-europeo, devono sottostare alle regole europee in materia di concorrenza ed alle regole comuni a tutti gli stati membri UE in tema di esportazione di tecnologie “dual-use"25. L'interazione tra le competenze prevalentemente civili dell'ESA ed i crescenti interessi dell'UE nell'impiego delle capacità militari di Galileo resta ancora in fase di sviluppo.

Questo spaccato costituisce sia un esempio di "competence creep"26 che un test delle capacità spaziali civili. Gli appalti pubblici sono quindi una pratica standard per l'implementazione delle politiche e dei programmi spaziali nazionali, dell'UE e dell'ESA. Tuttavia, essendo tali attività spaziali parti integranti di programmi di ricerca finanziati con fondi pubblici, pur dando luogo effettivamente ad attività commerciali, non hanno un formale scopo primario commerciale. Programmi come il citato GMES ${ }^{27}$

\footnotetext{
${ }^{25}$ Regolamento del Consiglio n. 428/2009/EC del 5 maggio 2009 (entrato in vigore il 27 agosto 2009), in GU L 134/1 del 2009, per la costituzione di un regime comunitario per il controllo delle esportazioni, del trasporto e del transito di oggetti dal duplice utilizzo.

26 Da intendersi anche nel senso di una erosione o attrazione della competenza, intesa come lento "rosicchiamento" volto ad un trasferimento di competenze invisibile ed indolore.

${ }^{27}$ Cit. nota 5. Esso poneva le basi per la Climate Change Initiative, mediante l'archivio, produzione e valutazione dei dati essenziali sul clima. COPERNICUS è guidato dalla Commissione Europea in cooperazione con ESA e con l'Agenzia Europea per l'Ambiente, ed è stato ottimizzato per l'elaborazione di servizi informativi geo-spaziali per l'ambiente e della sicurezza cui i governi degli Stati membri ESA ed UE possono accedere in via autonoma ed indipendente. Il sistema monitora le condizioni del pianeta
} 
(oggi COPERNICUS), ad esempio, potranno portare allo sviluppo di opportunità di business una volta che i finanziamenti UE saranno passati da modelli di ricerca “orientati” a modelli di libero business commerciale ${ }^{28}$.

In sostanza, il diritto della concorrenza regola la gestione dei progetti spaziali e la partecipazione ad essi, nella misura in cui presidia un'equa ripartizione degli interessi nei vari mercati, tra i quali, il trasporto spaziale, i sistemi satellitari e i servizi spaziali.

\section{LA DISCIPLINA DELLA CONCORRENZA TRA UE E STATI MEMBRI}

Sebbene vi sia tradizionalmente un alto grado di cooperazione nella supervisione e nell'applicazione del diritto della concorrenza tra Stati membri e la Commissione europea $^{29}$, tuttavia, la sua applicazione al settore spaziale a livello UE è, ad eccezione del progetto Galileo, ancora nella sua fase iniziale.

L'accesso alla particolare attività spaziale è determinato dalle regole che disciplinano gli appalti pubblici, a prescindere dal fatto che l'attività spaziale sia privata, pubblica o un ibrido. Le norme nazionali ed europee in materia di appalti pubblici sottopongono gli operatori tradizionali a varie forme di procedure competitive e a vari pre-requisiti. Le regole sono state poste in essere per garantire che le imprese partecipino all'appalto se sono chiamate ufficialmente a partecipare alla gara. Le norme di coordinamento degli appalti non possono, tuttavia, regolare i successivi vincoli contrattuali tra le parti nelle fasi successive, in particolare tra appaltatori e subappaltatori o tra le altre parti interessate, incidendo sull'accesso di questi ai risultati del progetto. Questo livello di accesso, uso e rilascio delle licenze può essere oggetto di un accordo separato che, a sua volta, può essere rilevante per le restrizioni alla concorrenza.

Terra e si concentra su sei settori principali (ambiente marino, terrestre, atmosfera, gestione delle emergenze, sicurezza e monitoraggio dei cambiamenti climatici) combinando i dati e le immagini ottenuti mediante satelliti e sensori aerei, nonché le stazioni di terra (i cosiddetti dati "in situ”). Tra il 2014 e il 2030 si prevede che COPERNICUS (talvolta in altri idiomi lo si ritrova scritto "KOPERNIKUS”) apporti benefici quantificabili in oltre 70 miliardi di euro, oltre a rendere il pianeta un luogo più sicuro e più protetto dal punto di vista dell'analisi della sostenibilità delle attività umane.

${ }^{28} \mathrm{Si}$ veda il citato Regolamento del Parlamento europeo e del Consiglio sul programma europeo di monitoraggio sulla Terra (GMES), n. 911/2010/EU del 22 settembre 2010.

29 La Commissione è l'istituzione europea espressamente delegata all'indagine, valutazione e sanzionamento delle violazioni della concorrenza. La diretta applicabilità delle citate disposizioni di cui agli articoli 101 e 102 del TFUE (ex articoli 81 e 82 del trattato CE) ha portato la Commissione europea nel 1980 a incoraggiare i governi nazionali a trasformare e adattare le disposizioni nazionali in materia di concorrenza. Questo processo è stato completato per tutti gli Stati membri. Si veda D. CAHILL, The Modernisation of EU Competition Law Enforcement in the EU, FIDE 2004 e H. ULLRICH, Evolution of European Competition Law, Whose Regulation, Which Competition, in ASCOLA Competition Law series, Edward Elgar Publ., Cheltenham, 2006. Si veda, per altro verso, il Regolamento 1/2003, entrato in vigore il 1 gennaio 2004, che assicura il coordinamento nell'applicazione del diritto della concorrenza europeo tra livello europeo e nazionale. Ciò crea competenze regolamentari parallele tra una o più autorità nazionali. Infatti, a seguito della riforma procedurale e amministrativa, in particolare con il decentramento dell'applicazione del diritto della concorrenza, che ha avuto luogo nel 2004, le “Autorità Nazionali Garanti della Concorrenza” sono state da allora dotate del potere di applicare le norme europee sulla concorrenza a livello nazionale. 
Come detto, le disposizioni principali del diritto della concorrenza possono essere dichiarate in certe situazioni inapplicabili in forza dei regolamenti di esenzione per categoria ai sensi dell'articolo 101 (3) TFUE. Queste esenzioni limitano l'impatto degli accordi sleali o restrittivi che potrebbero altrimenti essere considerati giustificabili, ad esempio, per proteggere il trasferimento di tecnologia. $\mathrm{Ci}$ possono essere ragioni economiche imperative per la protezione e la limitazione dell'accesso al know-how e alla proprietà intellettuale che sottopongono le parti a necessarie restrizioni. Tali eccezioni evidenziano la logica dietro le regole che permettono agli aiuti di Stato di essere ammessi: vi può essere una chiara necessità di tutelare gli interessi nazionali, i quali, nel settore dello spazio, possono essere i risultati ottenuti da ricerca e sviluppo ad alto impatto tecnologico ${ }^{30}$.

\section{LE AUTORIZZAZIONI E LE CONCESSIONI DI LICENZE SPAZIALI IN RAPPORTO AL DIRITTO DELLA CONCORRENZA}

Altro aspetto specifico della disciplina della concorrenza per le attività spaziali riguarda le autorizzazioni e le concessioni di licenze in rapporto all'applicazione delle regole di concorrenza.

A tal proposito, se è vero, per un verso, che la concessione di licenze prevede e dovrebbe garantire che siano rispettati alcuni requisiti di legge (come quelli connessi alla regolazione della frequenza e al rispetto delle norme sulla responsabilità), per altro verso, la disciplina sulle intese anticoncorrenziali e sulla concorrenza sleale può essere, o meno, direttamente rilevante per il processo di autorizzazione. Ciò può dipendere dalla circostanza che la licenza attiene ad un'attività spaziale indipendente, come ad esempio un nuovo lancio, oppure che si tratta di un servizio che (in forza di disposizioni di de-regolazione) è già soggetto a norme in materia di appalti. Quest'ultimo caso si verifica quando l'accesso al mercato è limitato ad un certo numero di operatori oppure nel caso di operatori storici che già sono legalmente in possesso di licenze e di autorizzazioni nell'ambito di quel procedimento amministrativo. In sostanza, il rispetto delle regole di concorrenza può, ma non sempre deve, essere una pre-condizione per l'autorizzazione delle attività spaziali. Similmente, l'attività soggetta a licenza può, in alcuni casi, ma non necessariamente in tutti, sollevare questioni relative al diritto della concorrenza.

La normativa sui cartelli anticoncorrenziali, tuttavia, prescrive un esame a priori della posizione dell'impresa, in settori che sono soggetti a norme UE sugli appalti o che potrebbero creare monopoli economicamente ingiustificati. D'altra parte però, finché le attività ed i servizi spaziali europei riguardano gare d'appalto "complete” o "integrali”, come nel caso di GALILEO nell'Unione Europea, la concessione di licenze resta una questione amministrativa estranea ai procedimenti di valutazione di concentrazioni nel

\footnotetext{
${ }^{30}$ Regolamento n. CE 2659/2000 della Commissione del 29/ novembre 2000, sull'applicazione dell'art. 81(3) del Trattato, con particolare riferimento alla materia "Ricerca e Sviluppo". Si veda, inoltre, P. KOUTRAKOS, Regulation of Defence Products in the Law of the European Union, in "Trade, Foreign Policy and Defence in EU Constitutional law”, 2001, cap. 8.
} 
mercato. Invece, con riferimento ai mercati ed agli strumenti finanziari necessari a sviluppare servizi di navigazione e di osservazione della Terra, il rapporto tra normativa sui cartelli e licenze è più delicato. Ai sensi della disciplina sui cartelli, le agenzie spaziali, per aggiudicarsi la licenza, dovrebbero garantire che le loro autorizzazioni non creino situazioni anticoncorrenziali sul mercato.

Inoltre, la garanzia del rispetto di procedure di appalto formali sarebbe riposto nell'obbligo di trasparenza e di effettuare bandi di gara pubblici, ed ancora nell'obbligo, da parte degli enti regolatori e delle agenzie, di garantire pari opportunità e condizioni di accesso indipendentemente dalla dimensione del mercato. Peraltro, la particolare natura dei mercati nazionali connessi alla difesa (quindi ad esigenze di sicurezza) sembrerebbe già essere sufficiente a spiegare perché il settore dello spazio finora non abbia prodotto particolari forme di concentrazione o altre fattispecie che il diritto della concorrenza normalmente deve monitorare in altri mercati.

Ciononostante, dal punto di vista della concorrenza, il mercato delle attività spaziali sembrerebbe, invece, presentare monopoli o oligopoli in vari settori quali, innanzitutto, il lancio, ma anche la fornitura di singoli materiali o componenti, paradossalmente prodotti solo da piccole imprese ultra-specializzate ${ }^{31}$. A complicare questo quadro, come è noto, le attuali principali industrie spaziali presentano strutture aziendali integrate in complesse "holding", generalmente suddivise in differenti divisioni operative.

\section{L'IMPATTO DEL SISTEMA GALILEO}

Riepilogando, il settore spaziale sembrerebbe aver goduto finora, in vario modo, di una protezione dalla applicazione delle più rigorose prassi e regole antitrust. Da un lato, infatti, tale settore si è trincerato dietro il vessillo della ricerca (con la giustificazione che talune attività spaziali potenzialmente possono anche non dare mai luogo ad attività commerciali da tenere sotto controllo); dall'altro inoltre, anche qualora queste attività diano origine ad iniziative commerciali, molti accordi limitativi del libero mercato o semplicemente restrittivi dello sfruttamento dei risultati scientifici ottenuti possono come già detto - rientrare in un novero di esenzioni espressamente previste per il settore (difesa, sicurezza, pregresse concessioni o licenze).

Tuttavia, come indicato, l'industria spaziale sta evolvendo rapidamente ed i mercati legati allo spazio, tradizionalmente dominati da interessi nazionali di difesa e di sicurezza $^{32}$, dovranno confrontarsi necessariamente anche con i menzionati effetti della crescita dei programmi a livello $\mathrm{UE}^{33}$ e conseguentemente con il rafforzamento delle istanze di una più pressante vigilanza antitrust.

Verrebbe da dire che tali fenomeni bisognerebbe attenderseli proprio in conseguenza della piena operatività di iniziative epocali come quella del GNSS GALILEO e degli altri menzionati progetti spaziali europei di più alto profilo e maggiore impatto

\footnotetext{
${ }^{31}$ Inoltre, molti produttori e fornitori di specifici componenti, prodotti e servizi distribuiscono tramite società che rientrano nel novero delle piccole e medie imprese (PMI).

${ }^{32}$ Si veda W. VON KRIES, B. SCHMIDT-TEDD e K.U. SCHROGL, Grundzuge des Raumfahrtrechts, Rahmenbestimmungen und Anwendungsgebiete, 2002, cap. 15.

33 In particolare, nel settore delle reti trans-europee, nelle applicazioni ambientali all'interno dei programmi di osservazione della Terra (come GMES/COPERNICUS ed altre applicazioni connesse) ed il descritto mercato spaziale dei servizi secondari cui ambiscono molte PMI.
} 
economico. Questi infatti richiedono la conformità con le procedure di appalto dell'Unione che dipendono, a loro volta, dalle norme di bilancio UE applicabili. A queste problematiche si aggiunga quella accennata relativamente alle regole della Convenzione ESA sugli appalti per il settore spaziale le quali contrastano notevolmente con quelle dell'Unione Europea. Infatti, essendo queste fondate su una ratio di ritorno economico per gli Stati membri (proporzionale alle contribuzioni al bilancio dell'Organizzazione) e di promozione delle industrie nazionali, non sono progettate certo per favorire la libera concorrenza tra gli Stati membri come disciplinata dal diritto dell'Unione e conseguentemente dal diritto interno degli Stati membri.

\section{Le procedure d'appalto dell'ESA prima e dopo GALILEO}

Il sistema degli appalti ESA agisce nell'ambito e nei termini della Convenzione ESA. Come già descritto, le condizioni di approvvigionamento ESA hanno l'obiettivo di sostenere i programmi spaziali nazionali in proporzione ai contributi al bilancio da parte degli Stati membri. Questo permette efficacemente il sostegno diretto all'industria nazionale, un obiettivo che è contrario ai principi dell'Unione. Infatti, sebbene alcune forme di sovvenzioni statali possano essere dichiarate legittime ai sensi delle norme comunitarie sugli aiuti di Stato, queste restano un'eccezione rispetto alla regola, e non a caso richiedono la previa approvazione della Commissione europea. Al contrario, l'Agenzia spaziale europea applica una politica industriale che consente un chiaro sostegno per l'industria spaziale a livello nazionale ed europeo. Questo è reso possibile per il tramite dei cd. programmi spaziali facoltativi, i quali (è questa una delle principali differenze con i programmi obbligatori) rappresentano il principale strumento di "ritorno" verso gli Stati membri ${ }^{34}$.

L'approvvigionamento dell'ESA riflette tale particolare gerarchia industriale, lungo tutta la filiera, dal prime contractor fino al subappaltatore. L'ESA ha dunque il compito di monitorare la manutenzione delle varie fasi progettuali e seguirle fino al completamento. Questo è necessario per controllare, quanto più è possibile, i rischi che gravano sugli importanti investimenti impegnati nei progetti spaziali.

A difesa di questo sistema si è sostenuto che la concessione, su queste basi, di particolari progetti a favore dell'industria di singoli Stati membri, è stato l'unico elemento che ha potuto garantire lo sviluppo costante dell'industria spaziale europea, offrendo una contropartita per gli enormi rischi d'investimento. Peraltro, viene fatto notare che il sistema degli appalti ESA non esclude la possibilità di procedure in concorrenza aperta $^{35}$, mentre non è la libera concorrenza l'obiettivo primario, bensì, il successo dei suoi programmi che si ritiene passi per il sostegno alle industrie nazionali.

\footnotetext{
${ }^{34}$ Il ritorno per i programmi facoltativi è regolarmente monitorato ex art. IV, Allegato V, Convenzione ESA, n. 4-5. Ad integrazione di quanto già detto sull'argomento, si aggiunge che gli Stati membri si aggiudicano i contratti oltre che proporzionalmente al loro contributo finanziario, secondo un loro rispettivo coefficiente particolare. Questo è indicato come “principio del giusto ritorno" e ha dominato il funzionamento dei progetti spaziali dell'ESA fin dalla loro creazione.

${ }^{35}$ Ai sensi dell'articolo 10 (1.a) del regolamento sugli appalti dell'ESA, la trasparenza ed il trattamento giusto ed equo devono essere assicurati tra i competitors.
} 
$\mathrm{Al}$ riguardo, è importante rilevare che, anche se la gara d'appalto è quella principale, in base al nuovo regolamento sugli appalti dell'ESA ci sono generalmente ben cinque tipi di procedure ${ }^{36}$ di partecipazione ai bandi dell'ESA.

A difesa del sistema di procurement dell'ESA v'è anche il rilievo secondo cui la scelta sul tipo specifico di gara da adottare dipende - in particolare - dal progetto (o programma) in fase di sviluppo ed inoltre, che è il Comitato per la politica industriale (IPC) il responsabile della supervisione e si occupa di tutti i tipi di procedure di appalto, ma resta salva la facoltà dell'ESA di destinare alcuni progetti a piccole e medie imprese o istituti di ricerca ${ }^{37}$, escludendo quindi i grandi e principali operatori del sistema. Inoltre l'ESA, nel periodo in cui si è svolto il dibattito sulla sua riforma dietro le pressioni derivanti dall'avvento di GALILEO, ha spesso rivendicato l'evidenza storica dei suoi processi decisionali ${ }^{38}$ e la pubblica trasparenza ${ }^{39}$ delle sue "call for tender". Infatti, ad esempio, una volta che il contenuto della gara ESA - riportato in un manuale di valutazione delle offerte (TEM - Tender Evaluation Manual) - sia stato pubblicato e il Tender Evaluation Board (TEB) abbia garantito la regolarità della procedura, vi è un periodo durante il quale l'ESA e l'appaltatore continuano a negoziare su aspetti essenziali, anche se è già stata effettuata la scelta del contraente ${ }^{40}$. Non a caso, ad esempio, questa procedura ha costituito una delle peculiarità addotte a propria difesa dalla lobby ESA.

\section{La riforma delle norme sugli appalti ESA.}

L'Agenzia spaziale europea ha deciso, nel dicembre $2008^{41}$, di effettuare una revisione delle proprie norme sugli appalti al fine di introdurre una maggiore trasparenza. Di

\footnotetext{
${ }^{36}$ L'articolo 13 del regolamento sugli appalti prevede due sotto-categorie all'interno della gara d'appalto: la gara aperta e la gara ristretta. La prima è il tipo generale e più utilizzato di procedura di gara. La gara non competitiva, disciplinata dall'articolo 14 del regolamento sugli appalti, si verifica in particolari casi di appalti la cui soglia è inferiore a $€ 100.000$. L'articolo 15 del regolamento sugli appalti prevede un accordo quadro, che può essere utilizzato quando il progetto ha un budget limitato ed un arco temporale di massimo cinque anni. Infine, vi è la procedura in due fasi di cui all'articolo 16, destinata a facilitare progetti in cui la descrizione e la struttura non sono ancora stati messi a punto, richiedendone così un'ulteriore formulazione in una seconda fase. Quest'ultima categoria è particolarmente adatta ad essere utilizzata in caso di partnership pubblico-privata.

${ }^{37}$ Per perseguire uno sviluppo geograficamente armonico - se non uniforme - dell'industria spaziale europea e una diffusione armonica delle competenze e delle opportunità in tutti gli Stati membri.

${ }^{38}$ Le sue procedure, le clausole e le condizioni, comprese le norme per il subappalto e le best practices, si sono sviluppate a seguito di dibattito intergovernativo, guidato da interessi di coordinamento e finalizzato a perseguire gli obiettivi dei programmi spaziali europei e nazionali ai sensi dell'articolo II della convenzione ESA.

${ }^{39}$ I dettagli su come ESA opera nell'ambito dei suoi appalti sono facilmente accessibili tramite il suo sito web sotto la rubrica "fare affari con l'ESA". La procedura di appalto dell'ESA è soggetta alle singole norme contenute nel suo allegato V, come integrato dal suo regolamento interno, in base al particolare tipo gara.

${ }^{40}$ Da un punto di vista della concorrenza, la separazione tra l'ESA e regimi dell'UE, in tema di appalti e contratti nel settore spaziale, ha permesso ai programmi spaziali dell'ESA di operare indipendentemente da eventuali vincoli derivanti dalla disciplina comunitaria in materia di concorrenza, appalti e servizi.

${ }^{41}$ Anche qui risulta evidente la conferma della tesi prospettata nel corso di questo lavoro, per cui le ultime spinte evolutive del settore spaziale europeo, che vedono il progetto GALILEO quale suo perno centrale, ed hanno posto le basi dell'attuale panorama, hanno il proprio fulcro nel periodo storico a cavallo tra il
} 
conseguenza, le nuove norme ${ }^{42}$ hanno portato ad una procedura di revisione che consente una seconda valutazione o revisione per motivi procedurali. Questo nuovo regolamento degli appalti è una risposta ad una serie di critiche ed ai suggerimenti avanzati nel corso del tempo per quanto riguarda la necessità di una maggiore trasparenza e di nuova valutazione nell'ambito del processo di approvvigionamento (il cd. procurement). A tal proposito si nota, in particolare, il ruolo del "Mediatore" il quale vigila sulle modalità con cui vengono selezionati i capocommessa (primi subappaltatori $)^{43}$.

Il nuovo regolamento ESA sugli appalti è stato progettato per garantire una procedura corretta e il mantenimento di standard procedurali equi. Si noti, però, che le decisioni prese in questo ambito dall'ESA non sono sottoposte a controllo giurisdizionale, in quanto, quale Organizzazione internazionale, essa è immune dalla giurisdizione $e^{44}$.

Riepilogando, le differenze essenziali tra la procedura di appalto dell'ESA e quella dell'Unione Europea sono le seguenti: l'Unione Europea a prima vista impone rigorose formalità sostanziali cui l'ESA, neanche con la menzionata riforma, lontanamente si conforma. Da un punto di vista del diritto della concorrenza, inoltre, il principio ESA del giusto ritorno è in contrasto con il principio del mercato unico europeo in condizioni competitive paritarie e di sostanziale concorrenza. Non in ultimo, perché maggiore è il numero degli Stati membri all'interno dell'Unione, maggiore è il livello di ingresso per i potenziali concorrenti sul mercato specifico. Ciò vale ancora di più in vista dell'applicabilità dell'accordo OMC sugli appalti pubblici per la partecipazione di paesi terzi in programmi non legati alla sicurezza. Peraltro, permane una differenza procedurale impensabile nel sistema dell'Unione (ed in tutti gli ordinamenti nazionali che hanno regolamentato la concorrenza), quella della "rinegoziazione delle condizioni contrattuali dopo l'aggiudicazione della gara”.

\section{La dimensione internazionale del procurement e della procedura di appalto del GNSS Galileo.}

La decisione di localizzare GALILEO esattamente nelle competenze dell'Unione Europea è stata presa alla fine del 2007, dopo il fallimento del modello iniziale di partenariato pubblico-privato "PPP”. Posto dunque il progetto Galileo sotto l'egida

Progetto di Costituzione per l'Europa ed il Trattato di Lisbona, in cui mediante l'attribuzione di competenza spaziale all'Unione, l'UE ha potuto dare una ideale spallata alla difesa del sistema intergovernativo ESA, facendo leva su una più effettiva ed efficace attuazione dell' Accordo quadro tra le due Organizzazioni. In questo spaccato, ovviamente, essendovi una parziale identità di Stati membri in entrambe, un rilevante merito va attribuito alle Istituzioni europee - in particolare - alla Commissione, ed al Parlamento che l'ha a tratti sostenuta ed a tratti compulsata; entrambe favorendo, o forse condizionando, anche la convergenza del Consiglio.

${ }^{42}$ La Risoluzione "Procurement Regulations of the European Space Agency“ adottata dal Counsiglio ESA nel Dicembre 2008 (ESA/REG/001), suddivisa in sette parti: 1) general provisions and principles; 2) methods of procurement part 3) initiation of tender; 4) tendering proceedings; 5) award of contracts; 6) review; 7) amendements; e sei allegati relative alle istruzioni del Direttore Generale per l'attuazione.

${ }^{43}$ Il Mediatore non è, tuttavia, responsabile per le controversie tra l'industria e l'agenzia stessa, di cui è responsabile il prime contractor al quale devono essere notificati tutti questi problemi.

${ }^{44}$ Art. IV, Allegato I della Convenzione ESA. 
dell'Unione Europea, le procedure di acquisto per il segmento spaziale rientrano, conseguentemente ed automaticamente, nell'ambito del diritto dell'Unione Europea e delle sue disposizioni finanziarie in materia di appalti pubblici.

La base per le regole europee in materia degli appalti in questione si trova nel Regolamento del Consiglio n.1605 del 2002 relativo al bilancio dell'Unione ${ }^{45}$. Questo documento di principio è completato in modo più dettagliato dal Regolamento di esecuzione del bilancio dell'Unione n. 2342 del 2002, mentre le regole generali sul coordinamento delle modalità di appalto pubblico per gli Stati membri dell'UE erano allora contenute nelle note direttive del 2004, di recente novellate con l'introduzione della Direttiva del Parlamento UE n. 24 del $2014^{46}$ e già modificate in materia di aggiudicazione di commesse in ambito di difesa e sicurezza dalla Direttiva della Commissione n. 81 del $2009^{47}$. Tale regolamento contiene norme per l'attuazione e l'applicazione del bilancio generale dell'Unione Europea. Il Titolo V del regolamento di esecuzione specifica le disposizioni generali in materia di appalti, tra cui la procedura e i tipi di chiamate che possono essere effettuate. L'adesione europea all'Accordo generale OMC sugli appalti, di cui l'Unione è firmataria, dà luogo a ulteriori considerazioni sugli

\footnotetext{
${ }^{45}$ Regolamento (CE, Euratom) n. 1605/2002 del Consiglio, del 25 giugno 2002, che stabilisce il regolamento finanziario applicabile al bilancio generale delle Comunità europee.

${ }^{46}$ Le regole generali sul coordinamento delle modalità di appalto pubblico per gli Stati membri dell'Unione Europea erano contenute nelle Direttive n. 17/2004 e n. 18/2004, entrambe del 31 marzo 2004. Esse sono state superate dalle nuove direttive sugli appalti pubblici nei settori ordinari e speciali e nel settore delle concessioni e degli appalti pubblici in Europa (in GU UE L 94 del 28 marzo 2014): la direttiva del Parlamento europeo e del Consiglio, del 26 febbraio 2014 n. 2014/24/UE "sugli appalti pubblici" abroga la direttiva 2004/18/CE e la direttiva 2014/25/UE "sulle procedure d'appalto degli enti erogatori nei settori dell'acqua, dell'energia, dei trasporti e dei servizi postali" abroga la direttiva 2004/17/CE. Completamente innovativa è stata invece la direttiva 2014/23/UE "sull'aggiudicazione dei contratti di concessione". Tale intervento legislativo era parte delle 12 azioni prioritarie per migliorare il funzionamento del mercato unico, rappresentando un importante passo in avanti nella riforma degli appalti pubblici nell'UE con l'obiettivo di portare benefici in molti settori relativi alla fornitura di beni, opere e servizi. Le direttive recepivano anche il portato della giurisprudenza della Corte di Giustizia dell'UE e quindi hanno introdotto definizioni giuridiche più dettagliate per chiarire alcuni concetti di base, al fine di contribuire alla certezza del diritto. Tali direttive si rivolgono anche alla fase dell'esecuzione dell'appalto (subappalto, risoluzione e modifiche sostanziali che impongono di rinnovare il procedimento di gara): secondo il principio per cui le direttive possono spiegare il proprio effetto anche nella fase di esecuzione, con riferimento alla possibilità le condizioni del contratto possano essere modificate. Dai principi generali in materia di appalti pubblici deriva oggi un generale divieto di apportare modifiche oggettive e soggettive al contratto già concluso e in corso di esecuzione. Tale divieto va applicato anche al procedimento di aggiudicazione, dovendosi considerare le modifiche apportate alle disposizioni di un appalto pubblico in corso di validità come una nuova aggiudicazione, nel caso in cui, a seguito di dette modifiche, il contratto presenti caratteristiche sostanzialmente diverse rispetto a quelle dell'appalto iniziale. Le direttive appalti riflettono la consapevolezza del legislatore europeo circa la vulnerabilità alla corruzione del settore degli appalti, prevedendo specifiche disposizioni per la prevenzione della corruzione nelle procedure di affidamento degli appalti e concessioni.

${ }^{47}$ Direttiva 2009/81/CE del Parlamento europeo e del Consiglio del 13 luglio 2009 relativa al coordinamento delle procedure per l'aggiudicazione di taluni appalti di lavori, di forniture e di servizi nei settori della difesa e della sicurezza da parte delle amministrazioni aggiudicatrici e degli enti aggiudicatori, e recante modifica delle direttive 2004/17/CE e 2004/18/CE, in GU UE L 216/76 del 20/08/2009.
} 
ppalti relativi a GALILEO, in considerazione del fatto che l'Unione è vincolata di tal guisa vincolata dalla norme sugli appalti dell'OMC ${ }^{48}$.

All'ESA fu affidato il ruolo di ente appaltante nello sviluppo di GALILEO ${ }^{49}$, in modo che le successive fasi procedurali e amministrative potessero essere convogliate con successo attraverso i servizi di ESA e la sua divisione tecnica ESTEC. Il coinvolgimento dell'ESA era indispensabile per garantire l'attuazione della politica spaziale europea, che di fatto ha portato alla sua diretta gestione in tutto il processo di approvvigionamento.

La dimensione internazionale del procurement di Galileo è stato istituito anche in virtù della partecipazione europea all'OMC ${ }^{50}$. In tale contesto, tuttavia, le caratteristiche specifiche del settore spaziale, non in ultimo i suoi aspetti militari e di difesa, hanno portato a dubbi circa la possibile partecipazione di imprenditori extra-europei nella fase di realizzazione (a titolo oneroso) nell'ambito del procurement (esponendo per di più il settore spaziale europeo a una maggiore concorrenza extra-europea dannosa per il mercato interno UE). Tuttavia, come già sottolineato, lo stesso accordo sugli appalti pubblici prevede varie esenzioni dalle disposizioni in materia di appalti aperti, in particolare nel caso di interessi di sicurezza nazionale, come definito ai sensi dell'articolo 28 del citato accordo OMC; pertanto, data l'importanza della fornitura di servizi spaziali e delle attività relative al trasporto dell'energia e delle telecomunicazioni, ha prevalso, rispetto ai menzionati dubbi, la tesi che l'accordo sugli appalti pubblici fosse inapplicabile. Per questo motivo, tali ulteriori disposizioni dell'accordo sugli appalti pubblici non sono entrati in funzione in relazione al progetto GALILEO.

\section{LE NOVITÀ SPERIMENTATE PER IL PROCUREMENT DEL SISTEMA GALILEO}

Il procurement per Galileo sottopone i partecipanti ai principi generali e alle disposizioni di diritto primario e derivato dell'UE che si sono sviluppate nel corso degli anni: la parità di trattamento, la non discriminazione, il riconoscimento reciproco, la proporzionalità e la trasparenza ${ }^{51}$. Ciò a sua volta impone alti standard procedurali e sostanziali. Vale la pena rilevare che la procedura di appalto scelta per GALILEO

\footnotetext{
${ }^{48}$ L'interazione tra il diritto UE della concorrenza con le regole proprie delle organizzazioni in materia di commercio e servizi (GATS) è diventato un problema per GALILEO, in particolare in materia di appalti pubblici. Il Consiglio, che agisce a nome dell’Unione Europea, ha approvato l'accordo sugli appalti pubblici con la decisione 94/800, entrata in vigore il $1^{\circ}$ gennaio 1996, dopo il 1994 Uruguay Round. Essa contiene un accordo sugli appalti pubblici che è applicabile e vincolante per gli Stati firmatari dell'OMC. L'accordo sugli appalti pubblici non comprende soltanto le merci, ma anche i servizi, ivi inclusa la produzione e la costruzione. Di conseguenza, l'accordo sugli appalti pubblici applicandosi agli appalti pubblici nel settore spaziale, si applica in particolare all'imponente programma di bandiera GALILEO che prevede la produzione di una costellazione di satelliti, i quali presuppongono altrettanti e più lanciatori, componenti, softwares ed una miriade di servizi accessori (per non parlare dei servizi e delle applicazioni che ne scaturiranno).

49 Si veda la Comunicazione della Commissione europea, Processing Galileo: Reprofiling the European GNSS Programmes.

${ }^{50}$ Vedi nota 48.

${ }^{51}$ Art. 1 (3) del Trattato sull’Unione Europea; art. 17(1), Regolamento 683/2008.
} 
divenne oggetto di negoziazione con l'Agenzia spaziale europea in qualità di ente appaltante, proprio in considerazione della particolare complessità del contratto in questione $^{52}$.

La competenza per il progetto Galileo è della Direzione generale per l'energia e i trasporti (DG TREN) e ulteriori informazioni sugli appalti, compresi gli aggiornamenti sulla chiamata, sono disponibili in tutte le fasi grazie ai suoi Servizi. Il bando di gara originale per Galileo (fase FOC) è stato annunciato dal Consiglio europeo e poi pubblicato, nel luglio 2007. Tale comunicazione contiene le descrizioni del lavoro richiesto (WPS), e successivamente dettagliato all'articolo 17(3) del principale regolamento Galileo GNSS 683/2008 ${ }^{53}$. L'Articolo 17(1) del regolamento 683/2008 garantisce l'applicazione delle norme sugli appalti pubblici dell'Unione alla gara per GALILEO. Al fine di evitare una posizione dominante tra le imprese, ogni lavoro appaltato viene suddiviso in due categorie. In primo luogo, secondo la norma contenuta all'articolo 17 (3.b), la pratica del prime contractor è stata limitata a due dei sei pacchetti principali di lavoro. Utilizzando il meccanismo noto come la regola del $40 \%$, l'Articolo 17(3.c) garantisce che almeno il 40\% del valore complessivo del lavoro venga subappaltato a società diverse dai primi. In questo modo una distribuzione ragionevole di interessi economici potrebbe essere garantita tra gli operatori del mercato, garantendo l'assegnazione alle imprese più piccole ${ }^{54}$, con evidenti progressi (sul piano dell'applicazione dei principi di libera e trasparente concorrenza e di limitazione delle posizioni dominanti con conseguente maggiore pluralità di soggetti concorrenti, etc.) in direzione dei principi dell'Unione.

In conclusione, l'applicazione della consolidata normativa antitrust dell'UE per gli appalti relativi al sistema GALILEO, secondo modalità operative prestabilite, orientate al miglior adattamento alle specificità del settore spaziale cui tale sistema appartiene, stanno ponendo in essere un precedente ed una best practice astrattamente in grado di condizionare le future scelte di governance della politica spaziale e della politica industriale del relativo settore. Le risultanze dell'attuale sperimentazione lungi dal poter essere tratte in via definitiva già oggigiorno, potranno essere esaminate solo nelle fasi conclusive delle procedure di appalto in questione. Le ulteriori riflessioni sulla portata sperimentale ed innovativa del tema trattato in questo lavoro sono rimesse in sede di conclusioni.

\section{Conclusioni}

Appaiono ormai chiare le diverse filosofie che stanno dietro ai sistemi di approvvigionamento all'interno dell'Unione Europea e dell'Agenzia spaziale europea:

\footnotetext{
52 Art. 18 del Regolamento 683/2008.

${ }^{53}$ Gli inviti ufficiali per il progetto Galileo sono stati pubblicati nel supplemento alla Gazzetta ufficiale sul sito appalti pubblici Tender Electronic Daily (TED), con le Commissioni Direzione generale dell'Energia e dei trasporti, come autore ufficiale della chiamata.

54 Le particolari considerazioni di sicurezza connesse a GALILEO incidono sulle condizioni di partecipazione alla relativa gara: le imprese candidate per essere ammesse devono avere sede in uno degli Stati membri dell'Unione Europea. Alcune eccezioni sono stati autorizzate e ciò ha permesso l'inclusione di subappaltatori di paesi terzi. I servizi, tuttavia, rimangono limitati a società stabilite nell'Unione Europea o dello Spazio economico europeo (SEE), in modo che solo le imprese che operano all'interno dei confini europei sono stati ammessi a partecipare all'appalto.
} 
nel nuovo settore spaziale UE, l'obiettivo ultimo del diritto della concorrenza è di sostenere l'attuazione della politica spaziale europea come sancita nel Trattato di Lisbona; lo schema degli appalti dell'ESA è, al contrario, orientato al mantenimento e al servizio delle esigenze prevalentemente industriali dei mercati nazionali degli Stati membri. Questa è la differenza fondamentale tra $\mathrm{i}$ due differenti sistemi di approvvigionamento. Entrambi i sistemi garantiscono forme di concorrenza, ma solo la procedura di appalto dell'Unione Europea assicura piena competitività sul mercato.

La distinzione tra concorrenza e competitività è importante per valutare se le aperture di mercato e il supporto dei sistemi di approvvigionamento possano favorire una concorrenza economicamente sostenibile o ragionevole tra operatori. La mancanza del riesame nell'ambito della procedura di appalto dell'ESA non sembrerebbe garantire quest'ultimo requisito, ed inoltre, mentre il regolamento sugli appalti ESA ha risposto alle critiche contro la sua procedura di appalto con l'introduzione di una maggiore trasparenza, non ha offerto, tuttavia, rimedi applicabili a procedure legali. Nonostante queste differenze, sia l'Unione Europea che l'Agenzia spaziale europea facilitano e sostengono i loro rispettivi mercati spaziali, sia pure mediante meccanismi diversi.

Nel richiamare la premessa di questo lavoro, sembra importante evidenziare che ormai il Diritto europeo della concorrenza si applica attualmente alle attività spaziali dell'UE in materia di ambiente, energia e trasporti ed in particolare, al momento solo ai programmi di bandiera come, GALILEO (quindi anche EGNOS ${ }^{55}$ oltre che il citato GMES/COPERNICUS ${ }^{56}$ ). Di qui, posto che il procurement del sistema GALILEO è il primo vero banco di prova sperimentale dell'estensione delle norme antitrust UE in questo settore, la dottrina ancora non si è espressa circa le proiezioni, rectius i pronostici, sulle evoluzioni future che quest'esperienza produrrà. Dall'altra anche la giurisprudenza della Corte di Giustizia dell’Unione Europea è ancora tutta da formarsi in materia ${ }^{57}$. In sostanza, pare che il dibattito verta principalmente sull'ipotesi che la lobby industriale spaziale operante in seno all'ESA riesca ad evitare che anche in tema di appalti e concessioni prosegua il graduale processo di intromissione della Commissione europea nel settore spaziale, il quale, peraltro, parrebbe aver ricevuto

55 EGNOS (“European Geostationary Navigation Overlay System”) è il precursore del sistema GALILEO, nato dall'Accordo tra la Comunità europea con l'ESA e l'Organizzazione per la sicurezza della navigazione europea (EUROCONTROL) fu ideato per funzionare in interoperabilità con i sistemi di navigazione satellitare statunitense (GPS) e Russo (GLONASS) e per potenziare GALILEO in futuro. Pertanto, ha il merito di aver introdotto il sistema di radionavigazione satellitare globale "GNSS” (Global Navigation Satellite System) nel territorio europeo, migliorandone precisione ed affidabilità e certificando la fonte del segnale. EGNOS va classificato tra i "sistemi regionali di potenziamento" dei GNSS.

${ }^{56}$ Si vedano al riguardo anche, l'art. 17 (procurement) e 18 (ESA) del Regolamento (CE) n. 683/2008 del Parlamento europeo e del Consiglio, del 9 luglio 2008, concernente il proseguimento dell'attuazione dei programmi europei di navigazione satellitare (EGNOS e Galileo); nonché l'art 6 del Regolamento (UE) n. 911/2010 del Parlamento europeo e del Consiglio, del 22 settembre 2010, relativo al programma europeo di monitoraggio della terra (GMES) e alla sua fase iniziale di operatività (2011-2013).

57 Sull'argomento si veda l'intervento in rappresentanza della CGUE "Perspective from the Court of Justice of the European Union” di Benjamin Cheynel al ECSL Practitioners' Forum "Space Governance in Europe -Regulation of Space Activities”, ECSL/ESA, Parigi, 27 marzo 2015: in cui si evidenzia che non vi sono giudizi in materia di politica spaziale europea o ad es. ricorsi in annullamento contro atti dell'UE adottati in applicazione dell'art. 189 TFUE e che gli unici due giudizi riguardanti l'ESA in generale riguardano la materia di marchi e brevetti (Galileo International Technology c. Commissione, T279/03, EU:T:2006:121; e Galileo International Technology c. OHIM-ESA e Commissione (GALILEO) (T-450/11, EU:T:2014:771). 
un'accelerazione dalla previsione di una espressa competenza sulla politica spaziale dell'Unione ai sensi dell'art.189 TFUE a seguito del Trattato di Lisbona.

In definitiva, anche se il settore spaziale ha potuto finora godere di una protezione dall'applicazione delle più rigorose regole antitrust e tutt'ora, nell'applicazione della competenza dell'Unione ai sensi dell'art. 189 TFUE, esso può dirsi al sicuro dal verificarsi della cd. preemption $^{58}$ a favore dell'UE nell'ambito della condivisione parallela con gli Stati membri, con il presente lavoro si è inteso paventare (seppur nella consapevolezza che la dottrina sull'argomento si sta rivelando cauta ed attendista) che attualmente si sta svolgendo una stagione della politica e dell'industria spaziale europea che potrebbe vivere ulteriori sviluppi a seguito dell'esperienza della procedura di gestione degli appalti per GALILEO, gestita dal GSO dell'Agenzia UE del GNSS europeo, con la conseguenza di incidere profondamente sul mercato interno dell'Unione, sulla governance multilivello (il citato cd. triangolo istituzionale StatiESA/agenzia spaziali nazionali-UE) nel settore spaziale e forse anche sulla stessa normativa della concorrenza dell’Unione.

\footnotetext{
58 In particolare, M. C. MUÑOZ RODRIGUEZ, El Tratado de Lisboa: la acentuación de los límites estatales a la política espacial europea, in El Tratado de Lisboa: la salida de la crisis constitucional, (jornadas de la Asociacion Española de Profesores de Derecho Internacional -AEPIDRI- Madrid 17,18 Dicembre 2007) 2008 Iustel, pagg. 309 e ss.. Sull'argomento si veda anche, A. ARENA, Il Principio della preemption in Diritto dell'Unione Europea: esercizio delle competenze e ricognizione delle antinomie tra Diritto derivato e Diritto Nazionale, Napoli, Editoriale Scientifica, 2013.
} 